\title{
Preface
}

\section{Gender Surgery: A Truly Multidisciplinary Field}

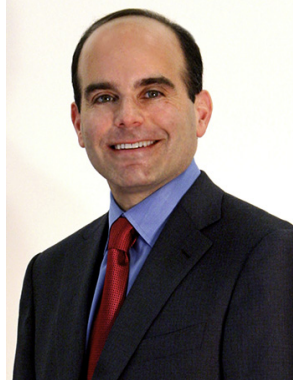

Loren S. Schechter, MD

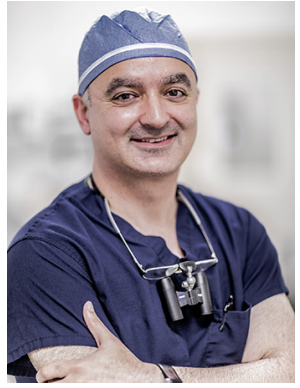

Bauback Safa, MD, MBA

Editors

Recent years have seen significant advances in the care of transgender and gender-diverse individuals. These advances have been timely as broadened insurance coverage has generated greatly increased demand for gender-affirming care, thereby exposing an inadequate supply of qualified practitioners. In order to help correct this discrepancy, much emphasis has been placed on practitioner education, spanning both surgical and nonsurgical specialties. For example, the World Professional Authority for Transgender Health initiated a Global Education Initiative, developing expert practitioners globally to better care for transgender and gender-diverse individuals. This educational initiative places an emphasis on multidisciplinary collaboration among primary care providers, endocrinologists, mental health providers, social workers, and surgeons of various specialties. Surgical societies have followed suit with greater inclusion of gender surgery-related topics in their respective annual meetings. This issue of Clinics in Plastic Surgery, dedicated entirely to care of the transgender and gender-diverse individuals, is one such example.

Transgender care requires a thorough and multisciplinary approach by experts in both surgical as well as nonsurgical fields. As such, it is imperative for any surgeon caring for transgender or diverse patients to be intimately familiar with the nonsurgical aspects of their care.

This issue of Clinics in Plastic Surgery has brought together a multidisciplinary group of experts in all aspects of transgender care to critically review current concepts in the field. Throughout the issue, we have attempted to cover aspects of transgender care that are pertinent to the surgical care of such patients, including medical, endocrine, and mental health aspects. We have also set out to give objective evidence for the currently available techniques in gender surgery. Our hope is for our readers to develop the skills to provide personalized, comprehensive, and multidisciplinary care for their patients, many of whom present unique circumstances, desires, and challenges during their transition. We are confident that this issue of Clinics in Plastic Surgery will be a valuable resource for surgeons faced with the complex care of transgender and gender-diverse individuals.

Loren S. Schechter, MD The Center for Gender Confirmation Weiss Memorial Hospital The University of Illinois at Chicago 9000 Waukegan Road, Suite 210 Morton Grove, IL 60053, USA

Bauback Safa, MD, MBA The Buncke Clinic

The San Francisco Transgender Institute 45 Castro Street, Suite 121 San Francisco, CA 94114, USA

E-mail addresses: drs@univplastics.com (L.S. Schechter) bauback@drsafa.com (B. Safa) 